\title{
EXPERIMENTAL STUDY OF VACUUM DIODES UNDER PARTIAL SPACE-CHARGE REGIME
}

\author{
J. A. N. Gonçalves, J. J. Barroso and G. M. Sandonato \\ Associated Plasma Laboratory, National Institute for Space Research \\ P. O. Box 515, São José dos Campos 12201-970, SP, Brazil
}

\begin{abstract}
Supported by analytical considerations and experimental verification, we demonstrate that thermionic electron current under partial space-charge condition follows the threehalves power Child-Langmuir law with a proportionality coefficient, $j$, that is material dependent and characterizes the emitter unambiguously. Such a partial space-charge regime has been assessed through experiments on parallel plane diodes with borondoped diamond films (with four different $\mathrm{B} / \mathrm{C}$ ratios) as electron emitters at temperature of $1000 \mathrm{~K}$. All current measurements have indicated a 3/2-power relationship on voltage but with distinct, material dependent coefficients $j$ that were found to decrease with the work function of the samples tested.
\end{abstract}

\section{Introduction}

The characteristics of diodes under conditions of complete space charge were calculated long time ago [1-2], and yet such a classical subject still remains as a source of refinement studies regularly appearing in the recent years [3-7]. In this regime, in which the space charge density is so high that the electric field at the cathode becomes zero, the emission current depends on the geometrical arrangement as well as on the applied potential, but not on the emission capabilities of the emitter. If the available emission, however, falls below the space charge limit, the current becomes dependent on the emission properties of the cathode as we shall see later on. It is this case which we refer to as the partial space charge condition, or the emission-limited condition. In many applications as in nanoemiters operating in the Schottky regime (in which the potential barrier at the emitter surface is lowered significantly by the cathode electric field) knowledge of the field actually existing at the cathode turns out necessary [8]. 
To investigate the partial space charge regime, we have performed experiments on a set of parallel plane diodes with the cathodes consisting of diamond films doped with boron at boron/carbon rations of 4,000, 8,000, 12,000, 20,000ppm. In the experiments, each cathode is heated up to $1000 \mathrm{~K}$ to produce thermal electrons and subjected to voltages in the $50-1000 \mathrm{~V}$ range.

Supported by analytical considerations, our results indicate that the measured currentvoltage characteristics follow the three-halves power Child-Langmuir law with a proportionality parameter that unambiguously identify each emitter tested. Unlike the space-charge-limited operation in which the current (irrespective of the emitter material) depends only on the applied voltage [9], in the observed regime of limited emission the current becomes material dependent, thereby rendering this regime useful at characterizing electron-emitting materials.

\section{Planar Diode under Emission-Limited Condition}

The following discussion is restricted to an infinite parallel-plane where the emitter is equipotential, relativistic effects do not enter, and initial velocities of the emitted particles are negligible. This latter assumption is not, however, a real limitation since the anode voltages employed in practical systems are so high that the initial energies of few tenths of an electron volt are indeed negligible. The fundamental equation governing the partial space-charge-limited flow are exactly the same as for the complete space-charge case, but with distinct boundary conditions. We begin with the energy conservation equation

$$
\frac{1}{2} m v^{2}=-e \phi(x)
$$

where $v$ is the electron velocity at the position $x, m$ and $e>0$ the mass and charge of the electron, and $\phi$ the classical electrostatic potential. In the inter electrode region, Poisson's equation determines the potential

$$
\nabla^{2} \phi=-\frac{\rho}{\varepsilon_{o}}
$$

where $\rho$ is charge density (negative in value for electrons) and $\varepsilon_{0}$ the electric constant (formally called the permittivity of free space). Closing the set of equations, we use the relationship that defines the electron current density 


$$
J=\rho v
$$

Combining (1) and (3) yields

$$
\left(\frac{d \phi}{d x}\right)^{2}=a^{2}+b \phi^{1 / 2}
$$

where $b=4 j / C$ with $C$ being a universal constant $C=\varepsilon_{o}(2 e / m)^{1 / 2}$; the integration constant $a$ is identified with the electric field $E_{\mathrm{o}}$ at the cathode trough the boundary condition $\phi(x=0)=0$, in correspondence with (1) where initial velocities have been assumed negligible. Integrating (4) by means of the substitution $u=\sqrt{a^{2}+b \phi^{1 / 2}}$ leads to

$$
\left(b V^{1 / 2}+a^{2}\right)^{1 / 2}\left(b V^{1 / 2}-2 a^{2}\right)-2 a^{3}=(3 / 4) b^{2} d
$$

where $V$ is the potential $\phi$ taken at the anode position $x=d$. Now defining the normalized quantities

$$
\begin{aligned}
& f=\frac{E_{o} d}{V} \\
& j=\frac{J}{J_{s c}}
\end{aligned}
$$

where

$$
J_{s c}=\frac{4}{9} \varepsilon_{o} \sqrt{\frac{2 e}{m}} \frac{V^{3 / 2}}{d^{2}}
$$

is the space-charge-limited current density, then (5) reduces to the quadratic equation

$$
j^{2}-j+(27 / 16) f^{2}(1-f)=0
$$

Thus when $f=0,(8)$ gives $j=1$ which from (6b) recovers the Child law [1] for the planar diode, and therefore, the normalized current density $j=1$ or its ajoint field parameter $f=0$ fully characterizes all space-charge-limited diodes. By contrast, in the other limit at $f=1$, i. e., the electric field at the cathode is the vacuum field $V / d$ itself, the associate root is $j=0$, meaning that there exists no space charge in the diode region. The general relationship $j(f)$ between the cathode electric field and the normalized current $j$ is plotted in Fig. 1. We see that the field decreases faster for currents close to complete space charge limitation. In fact, a 10 percent reduction of current from its saturation value (in which the electron field at the cathode is depressed to zero due to space charge) causes the emitter field to increase to about 25 percent of its value in the absence of space 
charge, whereas at $j=1 / 2$ (one-half of the saturation value) the field has decreased to only two-third of its vacuum-field value.

Thus we may conclude that even operating under partial space charge conditions, the diode current still scales with the three-halves power of the collector voltage, with the dependence of the cathode field on the current being given by (8) where the variable $j$ quantifies the magnitude of the space-charge effects as will be discussed in the next section.

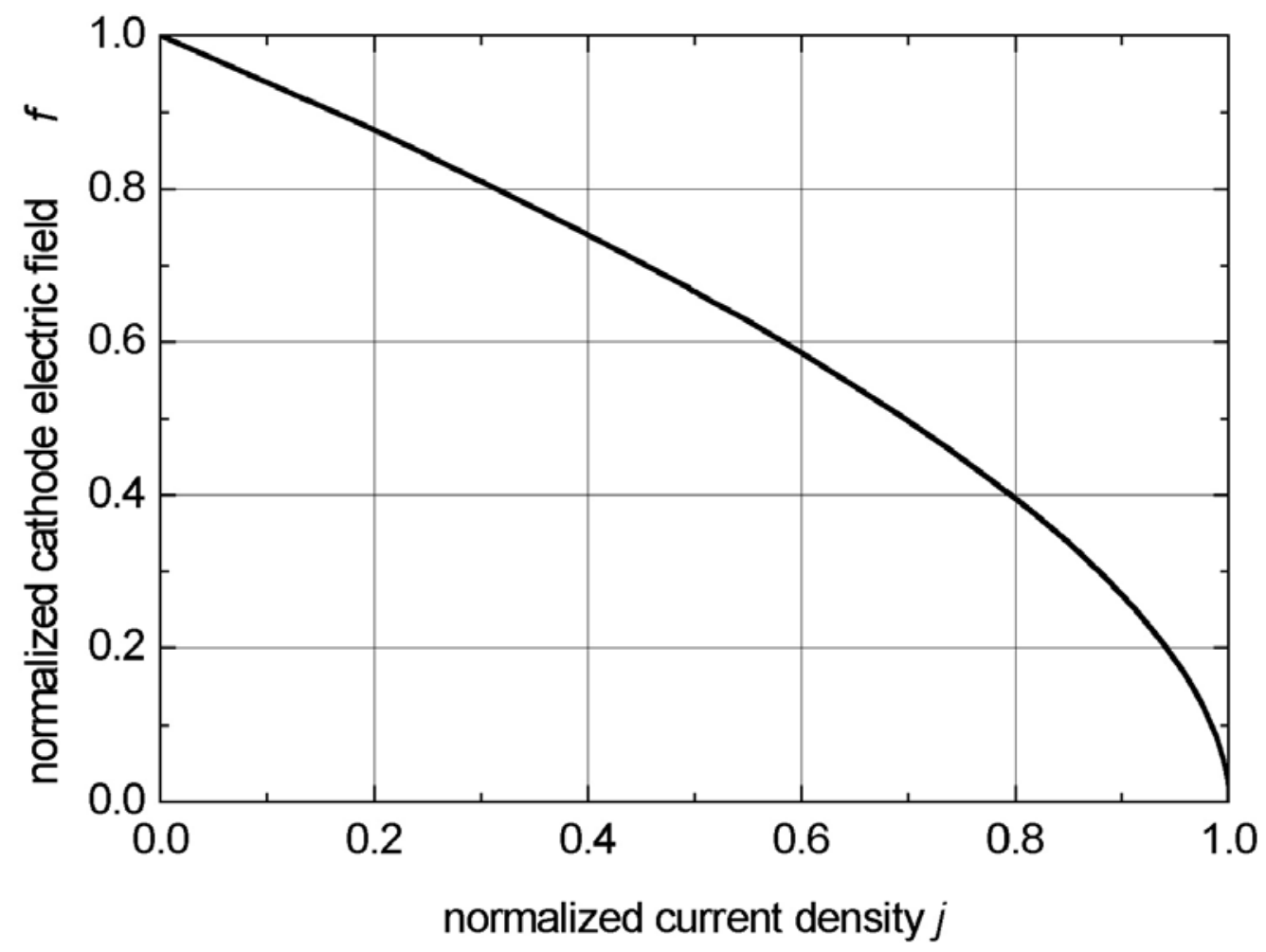

Fig. 1. Cathode electric field as function of current density (normalized quantities)

\section{Experiment}

The diamond films were grown on monocrystalline silicon (111) by hot filament CVD in a 10 -cm-diameter, $30 \mathrm{~cm}$ long cylindrical quartz reactor [10]. Boron was introduced into the reactor by flowing hydrogen through the $\mathrm{B}_{2} \mathrm{O}_{3}$ methanol solution. The substrates had been scratched by a diamond paste (grain size of $1 \mu \mathrm{m}$ ) to improve the nucleation of the diamond films, with growth parameters as listed in Table 1. 
Table 1 Growth parameters for the diamond films.

\begin{tabular}{|c|c|c|c|c|}
\hline & Sample 1 & Sample 2 & Sample 3 & Sample 4 \\
\hline $\mathrm{CH}_{4}(\mathrm{sccm})$ & 1 & 1 & 1 & 1 \\
\hline $\mathrm{H}_{2}(\mathrm{sccm})$ & 90 & 90 & 90 & 90 \\
\hline $\mathrm{B}_{2} \mathrm{O}_{3} / \mathrm{CH}_{3} \mathrm{OH} / \mathrm{H}_{2}(\mathrm{sccm})$ & 10 & 10 & 10 & 10 \\
\hline Temperature $\left({ }^{\circ} \mathrm{C}\right)$ & 50 & 50 & 50 & 50 \\
\hline Pressure (Torr) & 790 & 790 & 790 & 790 \\
\hline Time (h) & 6 & 6 & 6 & 6 \\
\hline $\mathrm{B} / \mathrm{C}(\mathrm{ppm})$ & 4,000 & 8,000 & 12,000 & 20,000 \\
\hline
\end{tabular}

The setup shown in Fig. 2 was used to measure the electron emission current of the diodes as function of the anode voltage. The diode was installed inside a cylindrical stainless steel chamber (diameter $15 \mathrm{~cm}$, length $45 \mathrm{~cm}$ ) connected to a diffusion vacuum pump that provided a base pressure of $10^{-7}$ mbar throughout the experiment.

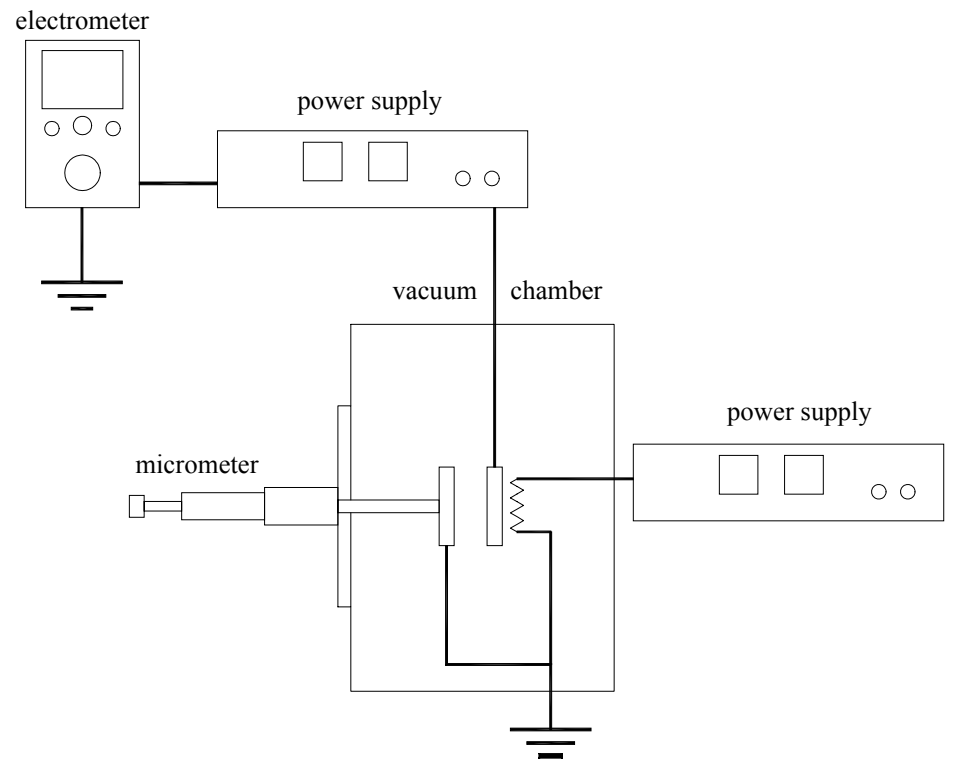

Fig. 2. Setup used to measure the electron emission current of the diodes as function of the anode voltage.

The circular cathode (of radius $10 \mathrm{~mm}$ ) was indirectly heated (at $1000 \mathrm{~K}$ ) by a coiled tungsten filament closely placed to the silicon substrate with the anode being polarized 
by a $2 \mathrm{kV} / 0.1 \mathrm{~A}$ DC power supply and spaced $d=1 \mathrm{~mm}$ apart from the cathode. A highsensitivity electrometer (able to detect currents as low as $10^{-13} \mathrm{~A}$ ) was used to measure the emission current upon increasing the anode voltage in steps of $50 \mathrm{~V}$.

Current-voltage measurements at $1000 \mathrm{~K}$ together with 3/2-power fitting curves are given in Fig. 3, where the experimental data follow the corresponding curve until their level off and saturate with voltage. We note that the measured currents are far below the level of currents emitter under complete space-charge limitation as predicted by Child's law (Fig. 4), which is associated with the proportionality coefficient $\mathrm{j}=4 / 9$. Instead, a diode operating under partial space-charge conditions is characterized by its own $j$ coefficient that identifies each cathode tested. To confirm this finding, we have determined the work function of each film. This has been done by measuring the emission current as function of temperature (at the anode voltage of $1000 \mathrm{~V}$ ) and then, on the basis of the Richardson equation for semiconductors [11], plotting $\mathrm{J} / \mathrm{T}^{5 / 4}$ versus $1 / \mathrm{T}$. A least-squares fit performed on each graph has given the work function values as listed in Table 2. We note that $j$ decreases with work function, and so this behavior suggests that thermionic emission in the partial space-charge regime is material dependent.

Table 2. Current fraction $j$ and work function of the samples tested.

\begin{tabular}{|c|c|c|}
\hline $\mathrm{B} / \mathrm{C}(\mathrm{ppm})$ & $\mathrm{j}$ & work function $(\mathrm{eV})$ \\
\hline 4,000 & $7.19 \times 10^{-7}$ & 3.01 \\
\hline 8,000 & $5.50 \times 10^{-7}$ & 3.23 \\
\hline 12,000 & $6.08 \times 10^{-8}$ & 3.44 \\
\hline 20,000 & $2.02 \times 10^{-7}$ & 3.29 \\
\hline
\end{tabular}




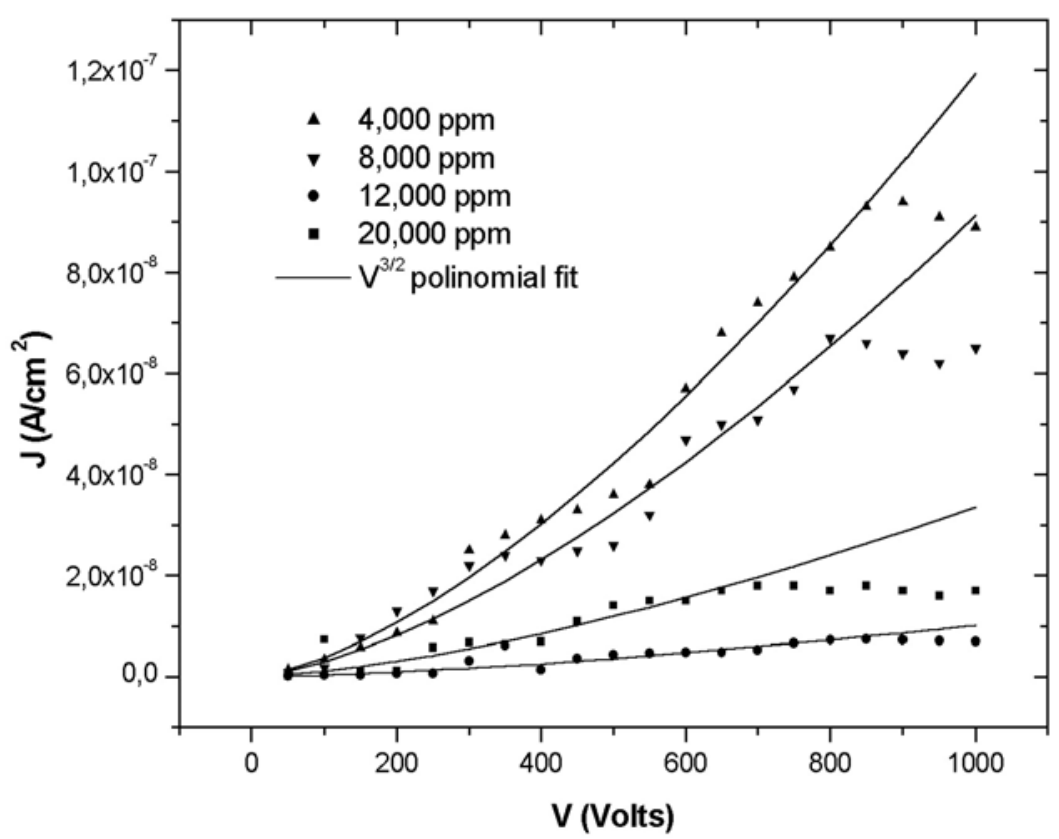

Fig. 3. Current-voltage measurements at $1000 \mathrm{~K}$

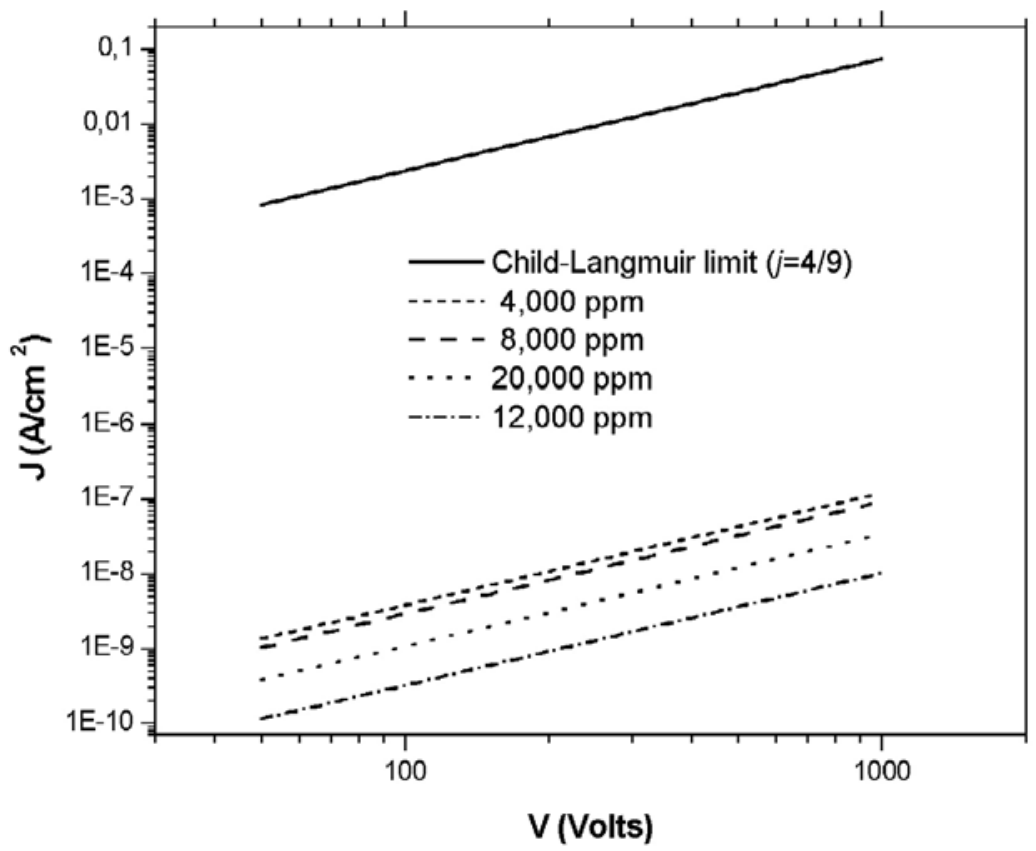

Fig. 4. Current densities measured under partial space-charge regime compared with the Langmuir-Child limit. 


\section{Conclusion}

Supported by analytical considerations and experimental verification, we have demonstrated that thermionic electron current under partial space-charge condition follows the three-halves power Child-Langmuir law with a proportionality coefficient $j$, that is material dependent and unambiguously characterizes the emitter. This differs from the space-charge-limited regime (in which more electrons are emitted than reach the anode) as identified by the normalized current density $j=1$ for planar diodes.

Such a partial space-charge regime has been assessed through experiments at $1000 \mathrm{~K}$ on parallel plane diodes with boron-doped diamond films (with different $\mathrm{B} / \mathrm{C}$ ratios) as electron emitters. Although six orders of magnitude below the full space-charge-limited current, the emission current measurements have indicated a 3/2-power dependence on voltage but with distinct, material dependent coefficients $j$ that were found to be a decreasing function of work function.

Therefore the experimental results indicate that under partial space-charge regime a given electron-emitting material can be identified by its current coefficient $j$, which thus becomes a useful parameter for materials characterization as far as electron emission properties are concerned.

\section{References}

[1] C. D. Child, “Discharge from hot CaO”, Phys. Rev., 32, $492-511$ (1911).

[2] I. Langmuir, "The effect of space charge and residual gases on thermionic currents in high vacuum", Phys. Rev., 2, 450-486 (1913).

[3] I. Langmuir and K. B. Blodgett, "Currents limited by space charge between coaxial cylinders", Phys. Rev., 22, 347-356 (1922).

[4] N. Jelic, R. Schrittwieser and S. Kuhn, "Revised generalized Child-Langmuir law", Phys. Lett., A246, 318-324 (1998).

[5] P. V. Akimov, H. Schamel, H. Kolinsky, A. Ya. Ender and V. I. Kuznetsov, Phys. of Plasmas, 8, 3788-3798, (2001).

[6] Y. Y. Lau, "Simple theory for the two-dimensional Child-Langmuir law", Phys. Rev. Lett., 87, 278301, (2001).

[7] K. G. Kostov and J. J. Barroso, "Space-charge-limeted current in cylindrical diodes with finite-length emitter", Phys. of Plasmas, 9, 1039-1042, (2002). 
[8] K. L Jensen, M. A. Kodis, R. A. Murphy and E. G. Zaidman, "Space charge effects on the current-voltage characteristics of gated field emitter arrays", J. Appl. Phys., 82(2), 845-954, (1997).

[9] J. Hasker and P A. M. van Dorst, "Pitfalls in the evaluation of cathode properties from I-V characteristics ”, IEEE Trans. Electron Devices, 36(1), 201-207, (1989).

[10] J. A. N. Gonçalves, G. M. Sandonato and K. Iha, "Characterization of boron doped CVD diamond films by Raman spectroscopy and X-ray diffractometry", Diamond and Rel. Mat., 11, 1578-1583, (2002).

[11] - Eisentein A. S., "Oxide Coated Cathodes", in Advances Electronics, Edited by L. Marton, Academic Press, New York, 1948, vol. 1,1-64, 1948. 